\title{
Mechatronic Design Optimization of a Teleoperation System based on Bounded Environment Passivity
}

\author{
Bert Willaert, Brecht Corteville, Herman Bruyninckx, Hendrik Van Brussel \\ and Emmanuel B. Vander Poorten \\ Dept. of Mechanical Engineering, K.U.Leuven \\ Celestijnenlaan 300B, 3001 Heverlee \\ bert.willaert@mech.kuleuven. be
}

\begin{abstract}
This paper presents a mechatronic analysis of a teleoperation system that provides haptic feedback. The analysis, based on the Bounded Environment Passivity method, describes the combined effect of the teleoperation controller and the master and slave hardware, including the effect of structural flexibilities, which have often been neglected in the domain of teleoperation. This results in rules of thumb for the design of the hardware and the tuning of the controller. As two of these rules of thumb pose a trade-off in hardware design, an unprecedented conceptual design optimization is described in a second part of this paper. The objective is to demonstrate the need for a mechatronic approach to the design of teleoperation systems in order to obtain maximal system performance.
\end{abstract}

Key words: Mechatronics - Teleoperation - Passivity

\section{Introduction}

In literature, most stability analyses of teleoperation systems with a bilateral controller assume rigid body dynamics for both master and slave robot 122345 . However, typical master and slave robots, especially the multi-d.o.f. systems, possess non-negligible flexibilities. Although the importance of structural flexibilities is well recognized in general [6], the effect of such flexibilities has received little attention in the domain of teleoperation. In 77, the closed-loop stability is analysed of teleoperation systems with a flexible slave. The generality of the obtained results, however, is restricted, as a fixed human operator is assumed and stability is quantified by time delay robustness. In [8], for teleoperation systems with a flexible slave, absolute stability is calculated based on Lewellyn's criteria. Here, the generality of the results is restricted by the assumption of a perfect position controller, i.e. $K_{p} \rightarrow \infty$.

This paper presents a mechatronic analysis of a teleoperation system with a flexible slave and a realistic position controller, based on bounded environment passivity [5]. Passivity theory is widely used to analyse the stability properties of teleoperation systems interacting with a wide range of human operators and 
environments. Here, the analysis is done for a Position-Force teleoperation controller. In the first part of this paper (section 2 and 3), the teleoperation system is described and its passivity properties are derived for both a rigid and a flexible slave robot. These properties result in rules of thumb for the design of the slave robot and the tuning of the controller.

Such rules of thumb, however, can be conflicting. A classical example is the trade-off between the structural stiffness of a robot and the total mass of the robot. Consequently, these rules of thumb give rise to an optimization problem. Therefore, in the second part of this paper (section 4), a conceptual example of such a mechatronic optimization problem is addressed. This example clearly demonstrates the importance of a mechatronic approach to the design of teleoperation systems.

\section{A mechatronic system}

A teleoperation system is a good example of a mechatronic system as it consists of two electronically controlled mechanical devices. The controller used here is the Position-Force controller (the PD-F scheme in [5]) shown in Fig. 2(a). The following equations, describe the forces applied by the motors of the master and the slave:

$$
\begin{gathered}
\tau_{m}=-\lambda \cdot F_{e} \\
\tau_{s}=\left(K_{v} s+K_{p}\right) \cdot\left(\mu \cdot x_{m}-x_{s}\right) .
\end{gathered}
$$

The parameters $\mu$ and $\lambda$ are the position and force scaling factors. Note that, at the master side, a low-impedance-type device is assumed and pure open-loop force control is used.

\subsection{A rigid body slave}

The teleoperation system analyzed in subsection 3.1 consists of a 1-d.o.f rigid body master and slave. These rigid body models for master and slave obey the following equations of motion:

$$
\begin{gathered}
F_{h}+\tau_{m}=M_{m} \ddot{x}_{m}+B_{m} \dot{x}_{m}, \\
\tau_{s}-F_{e}=M_{s} \ddot{x}_{s}+B_{s} \dot{x}_{s}, \\
Z_{m}=M_{m} s+B_{m}, \quad Z_{s}=M_{s} s+B_{s},
\end{gathered}
$$

with $Z_{m}$ and $Z_{s}$ representing the rigid body impedances of the master and the slave robot. Remark that for a rigid body model the positions $x_{m}$ and $x_{s}$ (the positions of the motors) are equal to respectively $x_{h}$ and $x_{e}$ (the positions of the end-effectors). 
(a)

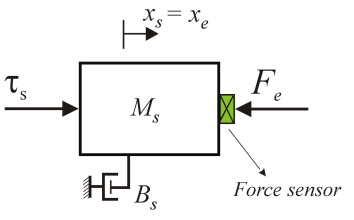

(b)

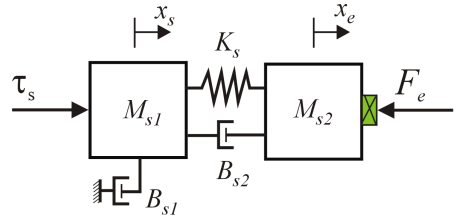

Fig. 1. A mechanical model for (a) a rigid body system and (b) a flexible system, represented by a fourth-order model.

\section{$2.2 \quad$ A flexible slave}

The teleoperation system analyzed in subsection 3.2 consists of a 1-d.o.f rigid body master and a fourth-order flexible slave. This fourth-order flexible slave obeys the following equation of motion:

$$
\left[\begin{array}{l}
\dot{x}_{s} \\
\dot{x}_{e}
\end{array}\right]=\left[\begin{array}{cc}
\frac{M_{s 2} s^{2}+B_{s 2} s+K_{s}}{\operatorname{Denum}(s)} & -\frac{\left(B_{s 2} s+K_{s}\right)}{\operatorname{Denum}(s)} \\
\frac{B_{s 2} s+K_{s}}{\operatorname{Denum}(s)} & -\frac{M_{s 1} s^{2}+\left(B_{s 1}+B_{s 2}\right) s+K_{s}}{\operatorname{Denum}(s)}
\end{array}\right] \cdot\left[\begin{array}{c}
\tau_{s} \\
F_{e}
\end{array}\right]
$$

with $\operatorname{Denum}(s)=M_{s 1} M_{s 2} s^{3}+\left(M_{s 2}\left(B_{s 1}+B_{s 2}\right)+M_{s 1} B_{s 2}\right) s^{2}+\left(\left(M_{s 1}+M_{s 2}\right) K_{s}+B_{s 1} B_{s 2}\right) s+K_{s} B_{s 1}$.

Both slave configurations are shown in Fig. 11. The rigid body parameters of the real 1-d.o.f. experimental master-slave setup described in [5], serve as a starting point for the calculations reported in this paper. Table 1 gives an overview of these parameters which are based on a linear model identification of the master and slave and the implementation of the controller on this setup.

Table 1. Parameters of the teleoperation system

\begin{tabular}{|lc|lc||l|l|l|}
\hline Model & & \multicolumn{3}{|l|}{ Controller } & \\
\hline \hline$M_{m}:$ & $0.64 \mathrm{~kg}$ & $B_{m}:$ & $3.4 \mathrm{Ns} / \mathrm{m}$ & $K_{p}: 4000 \mathrm{~N} / \mathrm{m}$ & $\mu:$ & 1 \\
$M_{s}:$ & $0.61 \mathrm{~kg}$ & $B_{s}:$ & $11 \mathrm{Ns} / \mathrm{m}$ & $K_{v}: 80 \mathrm{Ns} / \mathrm{m}(\zeta=0.81)$ & $\lambda:$ & 1 \\
\hline
\end{tabular}

\section{A mechatronic analysis}

This section describes a passivity analysis of the two teleoperation systems described above. The passivity analysis makes use of the bounded environment passivity method presented in [5]. This method is based on checking the positive realness of the admittance $Y_{M S}$, which represents the combined dynamics of the master, the slave, the controller and the environment. As can be seen in Fig. 2(b), this is the admittance the human operator interacts with. Passivity of this admittance $Y_{M S}$ is a sufficient condition for stable interaction between the teleoperation system and any (passive) human operator. The environment considered in this work is a pure spring $\left(K_{e}\right)$, because in the case of the PositionForce controller, stability problems are being reported especially for stiff environments 12 5. Moreover, the authors proved that a pure mass cannot make the admittance $Y_{M S}$ active for a rigid body slave 9 . 


\subsection{A rigid body slave}

For the teleoperation system described in subsection 2.1, having a rigid body slave, and assuming a pure stiffness as environment, the admittance $Y_{M S\left(K_{e}\right)}$ can be written as:

$$
Y_{M S\left(K_{e}\right)}=\frac{v_{h}}{F_{h}}=\frac{s\left(M_{s} s^{2}+\left(B_{s}+K_{v}\right) s+\left(K_{p}+K_{e}\right)\right)}{\left(M_{m} s^{2}+B_{m} s\right)\left(M_{s} s^{2}+\left(B_{s}+K_{v}\right) s+\left(K_{p}+K_{e}\right)\right)+\mu \lambda K_{e}\left(K_{v} s+K_{p}\right)},
$$

by combining (1), (2), (3) and (4). In [5, it is shown that the positive-realness condition for this $Y_{M S\left(K_{e}\right)}$ results in a complex set of conditions, that can be summarized as an upper boundary on the product of the scaling factors, $\mu \lambda$. Here, this boundary is rewritten as an upper boundary on the environment stiffness:

$$
K_{e} \leq \frac{B_{m}\left(B_{s}+K_{v}\right)}{\mu^{2} \lambda^{2} M_{s} K_{v}^{2}}\left(\mu \lambda\left(K_{v}\left(B_{s}+K_{v}\right)-2 M_{s} K_{p}\right)+2 B_{m}\left(B_{s}+K_{v}\right)+2 \sqrt{\text { Root }}\right)
$$

with Root $=\left(B_{m}^{2}+\mu \lambda B_{m} K_{v}\right)\left(K_{v}+B_{s}\right)^{2}-\mu \lambda M_{s} K_{p} B_{m}\left(K_{v}+B_{s}\right)+\mu^{2} \lambda^{2} M_{s} K_{p}\left(M_{s} K_{p}-B_{s} K_{v}\right)$.

Condition (8) specifies the maximum environment stiffness $K_{e}^{\max }$ for which the admittance $Y_{M S\left(K_{e}\right)}$ is passive as a function of all parameters of the system. Based on (8), Fig. 3 clearly shows the negative effect of the slave inertia $M_{s}$ and the positive effect of the stiffness of the position controller $K_{p}$ on the maximum environment stiffness $K_{e}^{\max }$. Note that both relations $M_{s}-K_{e}^{\max }$ and $K_{p}-K_{e}^{\max }$ shown here, suppose a fixed damping ratio $\zeta$ for the slave, i.e. $K_{v}=0.81$. $2 \sqrt{M_{s} K_{p}}$. Based on $(8)$, one can calculate that, for the parameters of Table 1 . the maximum environment stiffness for which $Y_{M S\left(K_{e}\right)}$ is passive is $580 \mathrm{~N} / \mathrm{m}$.

\subsection{A flexible slave}

For the teleoperation system described in subsection 2.2 i.e. with a flexible slave, the admittance $Y_{M S\left(K_{e}\right)}$ can be derived by combining (1), 22, (3) and (6):

$$
Y_{M S\left(K_{e}\right)}=\frac{v_{h}}{F_{h}}=\frac{N u m(s)}{\left(M_{m} s+B_{m}\right)(N u m(s))+\mu \lambda K_{e}\left(B_{s 2} s+K_{s}\right)\left(K_{v} s+K_{p}\right)}
$$

with $\operatorname{Num}(s)=\left(M_{s 1} s^{2}+\left(B_{s 1}+B_{s 2}+K_{v}\right) s+\left(K_{s}+K_{p}\right)\right) \cdot\left(M_{s 2} s^{2}+B_{s 2} s+\left(K_{s}+K_{e}\right)\right)-\left(B_{s 2} s+K_{s}\right)^{2}$.

The condition of positive realness for this admittance is too complex to be analyzed analytically. Therefore, the bounded environment passivity is analyzed numerically in Matlabß). For a set of fixed system parameters, $K_{e}^{\max }$ is found as follows: starting from $K_{e}=0$, the condition $\Re\left(Y_{M S\left(K_{e}\right)}(j \omega)\right) \geq 0$ is verified for $\omega \in\left[0.1-10^{9} \mathrm{rad} / \mathrm{s}\right]$ and for increasing values of $K_{e}$ (increment: $1 \mathrm{~N} / \mathrm{m}$ ). When the condition $\Re\left(Y_{M S\left(K_{e}\right)}(j \omega)\right) \geq 0$ no longer holds, $K_{e}^{\max }$ is obtained.

(a)

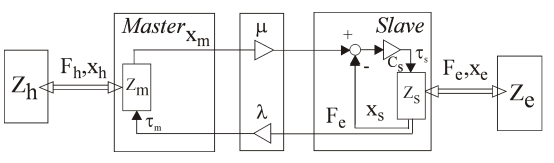

(b)

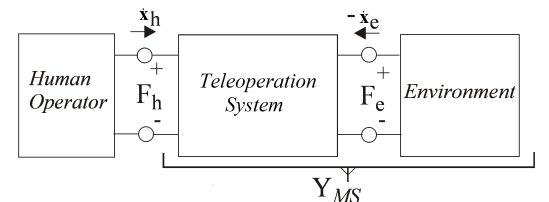

Fig. 2. (a) The controller representation and (b) the network representation of the analyzed teleoperation system. 


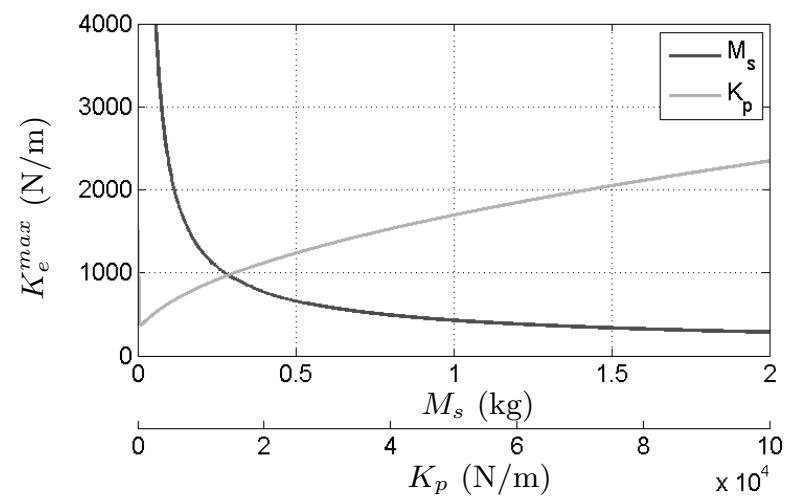

Fig. 3. The effect of the slave inertia $M_{s}$ and the stiffness of the position controller $K_{p}$ on the maximum environment stiffness $K_{e}^{\max }$, based on Bounded Environment Passivity.

First, the effect of the structural stiffness $K_{s}$ of the slave on the maximum environment stiffness $K_{e}^{\max }$ is analyzed. This is done for the parameters of Table $1\left(B_{s 1}=B_{s}\right.$ and $\left.B_{s 2}=2 \mathrm{Ns} / \mathrm{m}\right)$ and three different mass distributions, while the total mass of the slave is kept constant, i.e. $M_{s}=M_{s 1}+M_{s 2}=0.61 \mathrm{~kg}$. Fig. 4(a) shows the maximum environment stiffness $K_{e}^{\max }$ as a function of the structural stiffness of the slave, while Fig. 4(b) shows the same information but as a function of the structural anti-resonance frequency of the slave, $\omega_{s, a}$. One can see that for high structural anti-resonance frequencies $(>50 \mathrm{~Hz})$, the flexibility of the slave has little effect on the maximum environment stiffness, but for low structural anti-resonance frequencies $(<50 \mathrm{~Hz})$, the effect is very clear. One can see that for a structural stiffness going to infinity, $K_{e}^{\max }$ tends to $580 \mathrm{~N} / \mathrm{m}$, which is exactly the value found in section 3.1 for a rigid body slave (blue dashed line).

Next, in analogy of subsection 3.1 the effect of the controller stiffness, $K_{p}$, and the total mass of the slave, $\bar{M}_{s}$, is analyzed. Fig. 5(a) and Fig. 5(b) respectively show the maximum environment stiffness $K_{e}^{m a x}$ as a function of the controller stiffness of the slave, $K_{p}$, and as a function of the total mass of the slave, $M_{s}$. The calculation is done for five different values of the structural stiffness $K_{s}$ and the damping ratio for the slave is kept constant $(\zeta=0.81)$, i.e. $K_{v}$ is set to $0.81 \cdot 2 \sqrt{\left(M_{s 1}+M_{s 2}\right) K_{p}}$. In both figures, the curve for a rigid slave, derived in section 3.1, is also shown (blue dashed line). Based on classical control theory, one can assume that there is an optimal relation between the first structural anti-resonance frequency $\omega_{s, a}$ of the slave and the bandwidth of the position controller $\omega_{c}$. Fig. $5(\mathrm{a})$ confirms this assumption: it is clear that there is an optimal value for $K_{p}$ depending on the value of $K_{s}$. Here, it turns out that the optimal value for $K_{p}$ is $\pm \frac{1}{4} K_{s}$. Although the trend is general for the Position-Force controller, it is important to stress that this ratio is not, as it depends on all parameters of the system. Stated differently, one can say that 
(a)

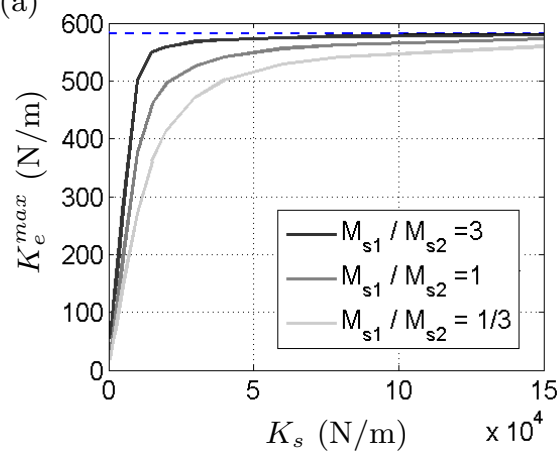

(b)

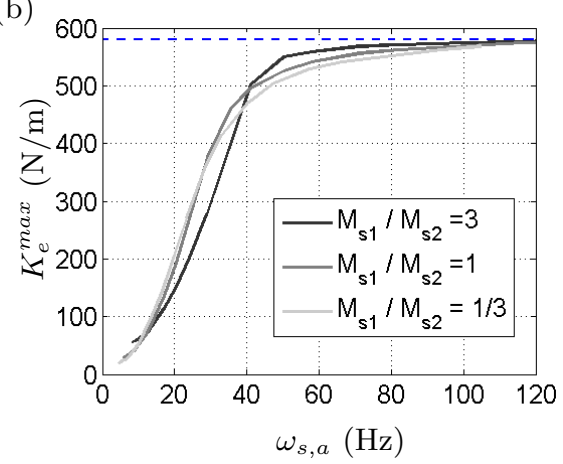

Fig. 4. The maximum environment stiffness for which $Y_{M S\left(K_{e}\right)}$ is passive as a function of (a) the structural stiffness $K_{s}$ of the slave and (b) the structural anti-resonance frequency, $\omega_{s, a}=\sqrt{\frac{K_{s}}{M_{s 2}}}$.

(a)

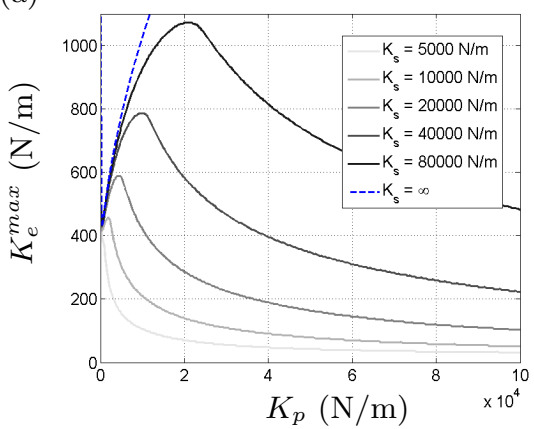

(b)

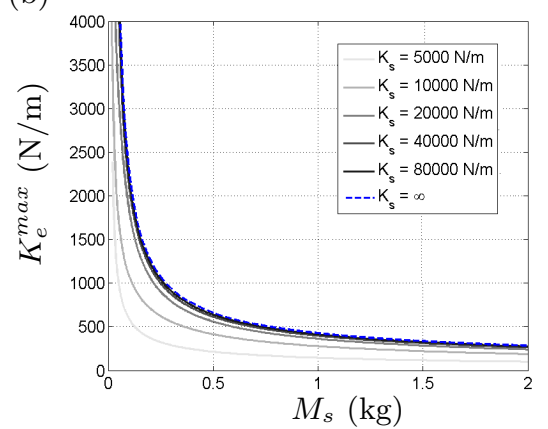

Fig. 5. The maximum environment stiffness for which $Y_{M S\left(K_{e}\right)}$ is passive as a function of (a) the controller stiffness $K_{p}$ and (b) the total mass $M_{s}$ of the slave.

for a maximal $K_{e}^{\max }$ the bandwidth of the position controller $\omega_{c}$ should be 'sufficiently smaller' than the first structural anti-resonance frequency $\omega_{s, a}$. Note that the effect of $K_{p}$ described here is a good example of the important fact that rules of thumb derived for a system with rigid body devices are not necessarily valid for a system with flexible devices.

\section{A mechatronic optimization.}

The previous section describes a mechatronic analysis of a 1-d.o.f teleoperation system resulting in a few rules of thumb, specific for the Position-force controller:

1. the slave robot should be as lightweight as possible, for a rigid body slave as well as for a flexible slave,

2. the structural stiffness of the slave robot should be as high a possible,

3. the stiffness of the position controller has an optimal value depending on the first structural resonance of the slave. 
The first and second rule of thumb pose a well-known and often appearing tradeoff in hardware design. Furthermore, it can be shown that this trade-off depends on the relation between the structural resonance of the slave and the bandwidth of the position controller. The relevance of these considerations is demonstrated through the following conceptual mechatronic design optimization.

Assume a slave robot consisting of two masses $\left(m_{1}=0.3\right.$ and $\left.m_{2}=0.2\right)$ connected through a link as shown in Fig. 6(a). The geometry of the link (length: $l=0.2 \mathrm{~m}$, rectangular section with thickness: $t=0.01 \mathrm{~m})$ and the material (Aluminium with $\rho: 2700 \mathrm{~kg} / \mathrm{m}^{3}$ and $E: 69 \mathrm{GPa}$ ) are fixed a priori, while the width of the rectangular section, $w$, is the parameter to be optimized. This conceptual design can be represented by a fourth-order model according to (6), with the following parameters:

$$
\begin{aligned}
M_{s 1} & =m_{1}+\frac{m_{\text {beam }}}{2} \text { and } \quad M_{s 2}=m_{2}+\frac{m_{\text {beam }}}{2} \quad\left(m_{\text {beam }}=\rho \cdot l \cdot t \cdot w\right) \\
K_{s} & =\frac{2 E I}{l^{3}} \quad\left(I=\frac{t \cdot w^{3}}{12}\right) \quad \text { and } \quad B_{s 2}=2 \mathrm{Ns} / \mathrm{m}
\end{aligned}
$$

As such, for increasing values of $w$, both the structural stiffness, $K_{s}$, and the mass of the slave, $M_{s}$, increase. Consequently, an optimal value for $w$ can be expected. Note that all other parameters, i.e. for the master and the controller, are the same as given in Table1. Now, for this teleoperation system, the Bounded Environment Passivity can be analyzed in the same way as described in section 3.2 . Fig. 6(b) shows the maximum environment stiffness $K_{e}^{\max }$ as a function of the geometrical parameter $w$, for five different values of the control parameter $K_{p}$. Again the damping ratio of the slave is kept constant $(\zeta=0.81)$. The following observations can be made:

- There is an optimal value for the geometrical parameter $w$. Initially, $K_{e}^{\max }$ increases for increasing values of $w$, which can be explained by the positive effect, shown in Fig. 4(a), of increasing the structural stiffness. However, Fig. 4(a) also shows that once the structural stiffness has reached a certain level, the benefit of further increasing $K_{s}$ is minimal. Therefore, $w$ reaches an optimal value at the moment the negative effect of the increasing total inertia $M_{s}$ becomes more dominant than the positive effect of the further increasing structural stiffness.

- The optimal value for the geometrical parameter $w$ depends on the controller stiffness $K_{p}$. In correspondence with the trend shown in Fig. $5(\mathrm{a})$, the optimal value for $w$, and thus the optimal structural stiffness, increases for increasing values of the controller stiffness $K_{p}$.

These observations underline the importance of a concurrent hardware and control design. How this should be done in practice for multi-d.o.f systems will be addressed in future research.

\section{Conclusions}

This paper presents a mechatronic analysis of the passivity of a 1-d.o.f. teleoperation system with a Position-force controller. Both a teleoperation system with a 
(a)

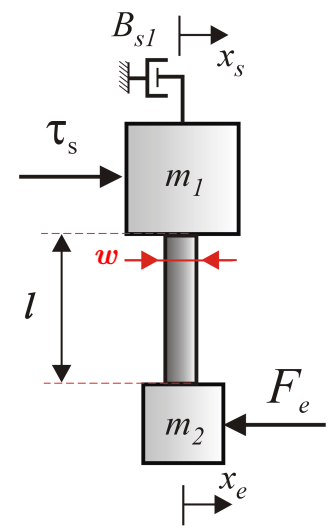

(b)

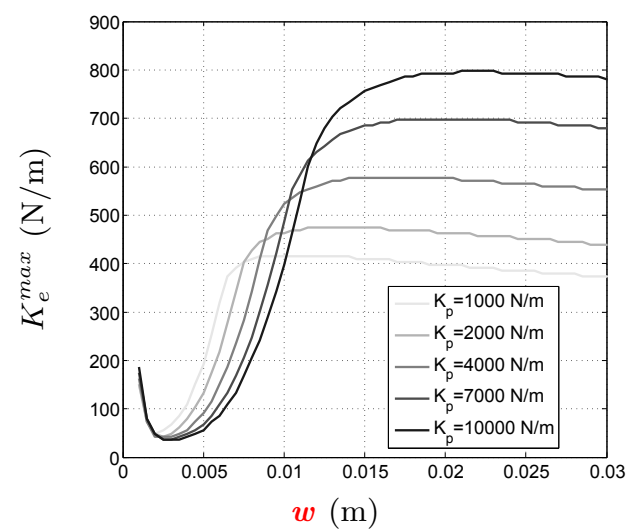

Fig. 6. (a) The conceptual design and (b) the maximum environment stiffness for which $Y_{M S\left(K_{e}\right)}$ is passive as a function of the geometrical parameter $w$.

rigid body slave and with a flexible slave are analyzed. The analysis demonstrates the need for a lightweight, structurally stiff slave robot. Another important conclusion from this analysis is the fact that rules of thumb for a system with rigid body devices are not necessarily valid for a system with flexible devices: in case of a rigid slave the position controller has to be as stiff as possible while for the case of a flexible slave, there is a maximum value for $K_{p}$ depending on the first structural resonance of the slave.

However, the above requirement for a lightweight, structurally stiff slave robot poses a trade-off in hardware design. Therefore, in a second part of this paper, a conceptual design optimization of a geometrical parameter of a robotic link is described. The objective of the optimization is to maximize the environment stiffness with which any passive human operator can stably interact. This conceptual optimization motivates a mechatronic approach to the design of teleoperation systems, i.e. a concurrent design of the hardware and the controller.

\section{Acknowledgments.}

This work was supported by a PhD grant from the Institute for the Promotion of Innovation through Science and Technology in Flanders (I.W.T.-Vlaanderen), two I.W.T projects, IWT/OZM/080086 and /080003, and by an FP7-People Marie Curie Reintegration Grant, PIRG03-2008-231045.

\section{References}

1. D.A. Lawrence. Stability and transparency in bilateral teleoperation. IEEE transactions on robotics and automation, 9(5):624-637, October 1993.

2. R.W. Daniel and P.R. McAree. Fundamental limits of performance for force reflecting teleoperation. The Int. J. of Robotics Research, 17(8):811-830, August 1998. 
3. K. Hashtrudi-Zaad and S.E. Salcudean. Analysis of control architectures for teleoperation systems with impedance/admittance master and slave manipulators. The Int. J. of Robotics Research, 20(6):419-445, June 2001.

4. E.B. Vander Poorten, Y. Yokokohji, and T. Yoshikawa. Stability analysis and robust control for fixed-scale teleoperation. Advanced Robotics, 20:681-706, 2006.

5. B. Willaert, B. Corteville, D. Reynaerts, H. Van Brussel, and E.B. Vander Poorten. Bounded environment passivity of the classical position-force teleoperation controller. In Proceedings of the IEEE/RSJ Int. Conference on Intelligent Robots and Systems, pages 4622-4628, St. Louis, MO, USA, October 2009.

6. S. K. Dwivedy and P. Eberhard. Dynamic analysis of flexible manipulators, a literature review. Mechanism and Machine Theory, 41:749777, 2006.

7. G.A.V. Christiansson and F.C.T. van der Helm. The low-stiffness teleoperator slave - a trade-off between stability and performance. The Int. J.l of Robotics Research, 26(3):287-299, March 2007.

8. M. Tavakoli and R.D. Howe. Haptic effect of surgical teleoperator flexibility. The Int. J. of Robotics Research, 28(10):1289-1302, October 2009.

9. B. Willaert, B. Corteville, D. Reynaerts, H. Van Brussel, and E.B. Vander Poorten. A mechatronic analysis of the classical position-force controller based on bounded environment passivity. Submitted to The Int. J. of Robotics Research. 